\title{
Manchas de tinta y autoconcepto del mexicano: relación etnopsicológica entre lo consciente y lo inconsciente
}

\author{
Joaquín Alberto Padilla-Bautista \\ Rolando Díaz-Loving \& \\ Isabel Reyes-Lagunes \\ Unidad de Investigaciones Psicosociales \\ Universidad Nacional Autónoma de México
}

Recibido: 23 de noviembre del 2012 / Aprobado: 28 de junio del 2013

Se analizaron los rasgos de personalidad de hombres y mujeres mediante la técnica de manchas de tinta de Holtzman y su relación con el autoconcepto. Los resultados reflejan la intensidad de los rasgos, ya que son expresados de una forma abierta y corroborados por el tipo de percepción que el proceso de socialización ha atribuido a cada sexo. Se puede apreciar, por un lado, la movilidad de las mujeres hacia una androginia y emancipación, y por otro, una permanencia de expresividad e instrumentalidad negativa, que denotan debilidad y vulnerabilidad. Los hombres comienzan a presentar características instrumentales más deseables para las mujeres.

percepción / manchas de tinta / instrumentalidad / expresividad / autoconcepto

Inkblots and Mexican self-concept: an etnopsychological relationship between the conscious and the unconscious

Personality traits of men and women were obtained through the Holtzman inkblot technique and related to self-concept, the results are a reflection of the intensity of these traits as they are expressed in an open way and are corroborated by the type of perception that the socialization process creates in each gender. On one hand the mobility of women towards androgyny and emancipation, and on the other a maintenance of negative expressiveness and instrumentality denoting weakness and vulnerability; while men begin to have more desirable instrumental features.

perception / inkblots / instrumentality / expressiveness / self-concept

Correo electrónico: loving@servidor.unam.mx 
El autoconcepto -al igual que la autoestima o la autoimagen- hace referencia a conceptos independientes, los cuales son conocidos como conceptos autorreferenciales empleados con frecuencia en relación con el self o sí mismo (Codina, 2005). El autoconcepto refiere a la descripción de todas las facetas y características que un individuo considera propias y que emplea para identificarse (Díaz-Guerrero \& Díaz-Loving, 1991). Es así que el autoconcepto es la creencia que tiene una persona de sí misma, es la imagen de sus habilidades y características, es entendido como un proceso psicológico cuyo dinamismo y contenido es determinado socialmente; engloba a un conjunto de percepciones, sentimientos, imágenes, autoatribuciones y juicios de valor referentes al propio individuo por lo que es, una realidad dinámica y activa de cada persona, por lo cual juega un papel decisivo en la conducta (Tamayo, 1982).

El autoconcepto es una de las variables más importantes de la personalidad, ya que permite entender el desarrollo y crear una imagen cada vez más estable de sí mismo, lo cual permite volverse más realista, tener un mejor conocimiento de las habilidades y limitaciones; lo que hace posible organizar el comportamiento (Shaffer, 2000).

Un aspecto fundamental para la formación del autoconcepto es el propio género, ya que este es una estructura cognitiva donde se asocia y organiza la información que percibe un indivi- duo y le indica desde pequeños cómo debe de ser un niño y una niña (Bem, 1981). El sexo, como indica Bem (1993), condiciona las interacciones sociales, el modo de actuar y de pensar que tenga una persona, y de esta forma es como las características biológicas en combinación con las enseñanzas impuestas por la sociedad condicionan los rasgos, los roles, las conductas y las ocupaciones, logrando como resultado el género y creando, asimismo, a hombres y mujeres.

Díaz-Guerrero (2003) reafirma esta posición ya que considera que las características asignadas a hombre y mujer son resultado de un proceso psicológico social y cultural, por lo que cada individuo se asume como perteneciente a un género en función de lo que cada cultura establece. Este proceso de socialización permite incorporar normas, reglas y expectativas; delimita comportamientos, características e incluso pensamientos y emociones que son adecuados para cada ser humano en base a esta red de estereotipos o ideas consensuadas.

Parsons y Bales (1955) han identificado la existencia de diferencias en el papel sexual; por un lado hablan de un papel instrumental y orientado a metas, el cual es asignado en la mayor parte de las sociedades a los hombres; y por otro, de un papel expresivo, orientado a las relaciones interpersonales, asignado con frecuencia a las mujeres. Bakan (1966, como se citó en Díaz-Loving, Rivera \& Sánchez, 2001) indica que 
existe una tendencia en los hombres a una diligencia y comunión, y por parte de las mujeres un sentido de abnegación que refiere a una preocupación por los demás y una necesidad de ellos. Sin embargo, estas dimensiones coexisten en mayor o en menor medida en la personalidad de todo individuo. Por lo que se puede decir que los dos sexos cuentan con la capacidad biológica para desarrollar cualquiera de estas conductas, quedando los papeles diferenciales de hombres y mujeres en los procesos de socialización.

Esta postura conceptual ha guiado el desarrollo de una teoría en la cual las características masculinas y femeninas se derivan del entrenamiento (proceso de socialización) que reciben hombres y mujeres en conductas instrumentales y expresivas. Por lo que para hablar de diferencias entre hombres y mujeres se deben considerar los elementos biológicos, papeles sociales y rasgos de personalidad como constructos que interactúan, pero ante todo como constructos independientes y mutuamente excluyentes (Díaz-Loving, Rivera \& Sánchez, 2001).

Díaz-Loving, Rivera y Sánchez (2001) enfatizan la importancia que tiene el estudio de estas dimensiones en cada grupo cultural y étnico al que se pertenece, así como al momento histórico y sociocultural particular de cada individuo, ya que la composición instrumental expresiva puede variar en aspectos deseables e indeseables tanto para los hombres como para las mujeres.

Este modelo replantea la existencia del modelo dualístico masculinidad/ feminidad donde se consideran como polos opuestos a cada uno de los sexos, y bajo esta nueva propuesta existe la posibilidad de encontrar a hombres y mujeres con elevados rasgos masculinos y femeninos, a los cuales se les conoce como andróginos. A estas personas se les considera sensibles y decididas a la vez, características que les permiten poseer una mayor capacidad de maniobra y flexibilidad en su comportamiento; existen también hombres y mujeres con altas características masculinas y bajas características femeninas, en los cuales se encuentra al estereotipo tradicional masculino; por otra parte, hay hombres y mujeres que muestran rasgos típicos femeninos y bajos rasgos masculinos, que son llamados femeninos; por último, se considera a hombres y mujeres como indiferenciados cuando muestran pocas características de personalidad asignadas socialmente a ambos géneros (Díaz-Loving, Rivera \& Sánchez, 2010).

De esta manera, al abordar el tema del autoconcepto se debe tomar en cuenta el proceso de socialización, pues al medirlo se recoge información del entrenamiento social al cual ha estado sometido el individuo. Actualmente, una de las formas como nos acercamos al autoconcepto de un individuo es mediante escalas autoaplicables, en las 
que el sujeto deposita en el instrumento las características que considera como suyas. Sin embargo, algunos teóricos como Wayne Holtzman refieren que la confiabilidad de los autoinventarios, y en especial los de personalidad, suele ser bastante alta, ya que tienen un autoconcepto consciente que controla sus respuestas a los test, el cual no está relacionado con la conducta social tal y como la juzgan otros (Holtzman, Thorpe, Swartz \& Herron, 1971).

Las escalas autoaplicables permiten al sujeto conocer cuál es el aspecto conductual que evaluará el calificador; de esta manera, estas escalas abren la posibilidad de dos interrogantes: ¿cómo saber si la información que nos brinda el sujeto carece de deseabilidad social?, y ¿cómo saber si el mecanismo de represión puede llegar a ocultar información ya sea de forma consciente o inconsciente? Pese a estas dudas las escalas de autoaplicacion siguen siendo un instrumento altamente empleado por investigadores, ya que la calificación que se hace de ellas se muestra confiable al eliminar la subjetividad de calificación del aplicador, la cual depende mucho de su propia experiencia y destreza. Por lo tanto, no se debe olvidar que la calificación de las escalas autoaplicables es una calificación objetiva de la información que se brinda; sin embargo, ya desde el principio la información brindada es de carácter subjetivo.

La subjetividad en un instrumento de medición que está asociado regu- larmente a las técnicas proyectivas empleadas dentro del área terapéutica; sin embargo, dentro del área organizacional estas han sido negadas en los estudios experimentales y sociales, al carecer de validez y confiablidad como instrumentos de medición. Cattell (1972) sostiene que estas pruebas son objetivas para la medición y observación, ya que no es posible falsear la información pues el sujeto responde sin saber cuál es el aspecto conductual que se evaluará. De la misma manera, hace hincapié en que es un error emplearlas solo como instrumentos de proyección por cuanto la calificación final depende en gran medida de la destreza de quien haga la interpretación, ya que esta puede variar de un calificador a otro.

Es así como se puede contar con dos tipos de medición obtenidas de un instrumento: las medidas objetivas, las cuales carecen de una manipulación consciente o inconsciente por parte del participante; y las medidas subjetivas, obtenidas con una posible manipulación y deseabilidad social. A la par, se cuenta con dos formas de calificación de esta misma medición: una objetiva, donde se sigue un procedimiento estandarizado de calificación que permite una calificación uniforme con amplia confiabilidad; y una calificación subjetiva, que depende de la propia habilidad y destreza del calificador.

Para esta investigación se empleó como instrumento de medición de la personalidad la técnica de manchas de 
tinta de Holtzman (HIT) (Holtzman et al., 1971), una técnica que como todos los instrumentos de personalidad trata de dar un panorama global del sujeto en lo que se refiere a su particular configuración de rasgos y conductas.

Como test proyectivo, el HIT logra penetrar en la personalidad individual y obtener respuestas de su mundo interno. Realiza un análisis cualitativo y posee a su vez características psicométricas para poder comparar y predecir de una forma cuantitativa, que lo hace un instrumento de gran importancia en el estudio de la personalidad (PadillaBautista, 2012). Los datos obtenidos del HIT son datos objetivos referidos por el participante, quien responde libremente sin conocer la interpretación y la manera como el administrador calificaría estos datos; se carece de una deseabilidad social, la cual es considerada por Matesanz (1997) como la tendencia que presentan los sujetos a idealizar sus respuestas según los estereotipos introducidos por la valoración social de deseabilidad. Es decir, se carece de una distorsión de las respuestas, por lo que el sujeto no puede falsear la información como mejor le convenga, y aproximarse así a las respuestas que son socialmente más deseables. Al ser de un carácter ambiguo, donde el sujeto no está enterado conscientemente de la situación, y tener que emplear su imaginación y creatividad, esta técnica tiende a rodear el proceso básico de represión
(Sha fer, 1954, como se citó en Vargas \& Villagómez, 2003).

El principal aspecto teórico de un instrumento como el HIT es el proceso de proyección, mediante el cual el sujeto percibe y da una interpretación a un estímulo o tarea ambigua, que revela aspectos importantes de su funcionamiento psicológico. Se puede asumir que la respuesta está basada en experiencias pasadas y que contienen información acerca de los sentimientos y las relaciones objetales del sujeto, por lo que en base a esto él debe organizar su respuesta basada en su propia personalidad y experiencia, y comunicarla. Para la interpretación es necesario tener en cuenta tres aspectos fundamentales de la personalidad, que permiten la comprensión y el significado de las respuestas del sujeto a la estimulación de las manchas de tinta: a) el estado emocional del individuo, b) los impulsos del individuo, y c) la suma del control de las reacciones internas. Las emociones corresponden a ciertos estados del cuerpo y las respuestas emocionales son diferenciadas por medio de la conducta. Experiencias como el placer o el displacer son el reflejo de reacciones internas; cuando una persona está estresada lo evidencia en su conducta, su inhabilidad para un buen desempeño de sus funciones, su nerviosismo; en una respiración entrecortada, en su cólera y en sus movimientos físicos. Algunos individuos que se encuentran dentro de una situación experimental se muestran 
conductualmente con cierta indiferencia, desean terminar pronto y con el menor involucramiento posible; otros, ante la tarea, se muestran con cierta curiosidad intelectual, deseando saber lo que pasará, ya que lo ven como un reto a su habilidad para desarrollar conceptos; y otros, en cambio, se muestran estresados, debido a la naturaleza ambigua del estímulo, ya que esto les genera tensión. Así, una mancha de tinta refleja la habilidad para controlar y dirigir sus reacciones cognitivas y afectivas, de acuerdo con las demandas contextuales y situacionales. Cuando un sujeto está funcionando adecuadamente, aun con un poco de confusión en su estado emocional, es capaz de controlar sus reacciones confortablemente, y facilita las percepciones que reflejan su estado interno. Por el contrario, en un estado de estrés el individuo no es capaz de controlar sus reacciones y sus percepciones están en función de sus necesidades y fantasías, lo cual revela confusión emocional, y no existe una conceptualización intelectual (Hill, como se citó en Vargas \& Villagómez, 2003).

Hill (como se citó en Vargas \& Villagómez, 2003), indica que algunos de los participantes toman como un reto la interpretación de las manchas y lo asumen como un ejercicio cognitivo, algunos más realizan el ejercicio debido a la necesidad de búsqueda, o encuentran en esta actividad un fin terapéutico. En general, estas personas son colaborado- ras y no ponen restricciones conscientes a sus respuestas. Sin embargo, otros sujetos ponen límites a sus respuestas y disminuyen su libertad de asociación; pueden carecer de motivación para cooperar o desean resistirse al examinador. Estas actitudes son un reflejo deliberado para sentir que las cosas se encuentran bien. Y tanto el número de rechazos como el tiempo de reacción reflejan la habilidad del sujeto para responder.

Tomando en cuenta que un individuo puede referir de forma consciente características que él considera como propias y, a la par, emitir de una manera inconsciente la información de su experiencia y personalidad.

¿Existe una relación entre lo que un individuo juzga de manera consciente con lo que manifiesta de una forma inconsciente? De ser así, ¿cuál es el grado de relación entre las características que el individuo considera como propias y las que puede manifestar bajo ciertas situaciones de la vida?

\section{MÉTOdO}

\section{Participantes}

La muestra no probabilística, por conveniencia, fue de 60 voluntarios (50\% hombres y $50 \%$ mujeres), entre los 19 y 26 años de edad ), todos ellos estudiantes de diferentes universidades. 


\section{Instrumentos}

Para la medición de rasgos de personalidad se empleó la forma A de las formas paralelas de la batería de manchas de tinta de Holtzman et al. (1971), conformada por 45 láminas, las cuales presentan una rica variedad de estímulos tanto cromáticos como acromáticos. Esto permite comprender los elementos que evocan la respuesta del sujeto mediante la calificación de 22 variables, que hacen posible captar la mayoría de las calificadas en el sistema Rorschach; sin embargo, permite una cuantificación unidimensional confiable de rasgos de personalidad. González (2006) refiere que la técnica Holtzman es considerada por algunos teóricos como una prueba especial, debido a que cuenta con las características de un instrumento proyectivo, pero permite su uso como un instrumento psicométrico, lo cual hace posible su uso en investigaciones sociales. Para la interpretación de las variables se toma como guía principal las propuestas por Hill (como se citó en Vargas \& Villagómez, 2003); este manual representa una explicación clínica del uso de las manchas de tinta de Holtzman.

Para la medición del autoconcepto se empleó la escala de Díaz-Loving, Reyes-Lagunes y Rivera (2002), un instrumento etnopsicológico para la medición del concepto del mexicano. Se conservaron los nueve factores originales, tomando solo los primeros cinco de cada uno de ellos: Social expresivo $(\mathrm{a}=.813)$, Ético normativo $(\mathrm{a}=.626)$, Inteligencia social emocional $(\mathrm{a}=.721)$, Control externo negativo pasivo $(\mathrm{a}=.825)$, Social afiliativo $(\mathrm{a}=.880)$, Emotivo negativo-autoafirmativo $(a=730)$, Instrumental constructivo $(a=.741)$, Vulnerabilidad emocional $(\mathrm{a}=.730)$ y Depresión $(\mathrm{a}=.775)$.

\section{Procedimiento}

La aplicación individual de la batería de pruebas se llevó a cabo dentro de cámaras Gessell y en salones de clase de cada una de las universidades, con una cita programada, se realizó un raport, que fue seguido de una entrevista breve; al concluir se contestó la escala de autoconcepto, y posteriormente se aplicaron las manchas de tinta.

\section{RESULTADOS}

Se procedió a la calificación del HIT conforme a la propuesta original del autor (Holtzman et al., 1971); de esta forma, las 22 dimensiones del HIT fueron calificadas en cada una de las 45 láminas de cada participante, obteniendo 990 puntuaciones para cada instrumento aplicado, lo que conformó un banco de datos de 59400 puntuaciones, las cuales fueron sumadas para cada una de sus variables, obteniendo calificaciones globales brutas para cada uno de los sujetos. Posteriormente se realizaron cómputos con los primeros cinco conceptos de cada factor de la escala de 
autoconcepto, obteniendo buenos índices de confiabilidad.

Para conocer si existe una relación con las 22 variables del HIT y los nueve factores de la escala de autoconcepto se empleó una correlación momento de Pearson para cada uno de los sexos, como se puede observar en la tabla 1 ; se obtuvieron 23 correlaciones para las mujeres y para los hombres únicamente dos, todos los coeficientes de correlación significativos tienen una consistencia teórica con las definiciones de los instrumentos empleados.

Las variables Tiempo de reacción, Verbalización patognómica, Abstracto, Penetración, Balance y Popular no mostraron correlación con algún factor de la escala de autoconcepto ni para hombres ni para mujeres.

De la misma manera, ninguno de los dos sexos presenta, para el factor social afiliativo de la escala de autoconcepto, correlación con alguna variable del HIT.

\section{Correlaciones obtenidas para las mujeres}

El factor social expresivo se refiere a individuos que cuentan con características positivas, las cuales les posibilitan una comunicación y expresión en su medio social. Estos individuos se muestran, en su relación con las variables del HIT, con una baja aptitud para reestructurar un campo perceptivo complejo, es de- cir, como dependientes de campo, con una necesidad de amistad, sensibles a otros, les afecta el estrés (Localización, $[\mathrm{r}=.-481, \mathrm{p}>.01])$. Tienen una capacidad para analizar un material en sus componentes y la relación de estos, lo cual requiere un esfuerzo intelectual; poseen un pensamiento realista, lógico, y una organización cognitiva que les permite emplear su fantasía (Integración $[\mathrm{r}=.461, \mathrm{p}>.05])$. El uso de estos recursos intelectuales les permite contar con una espontaneidad, lo cual está considerado como contrario al pensamiento estereotipado (Animal, [r=.389, $\mathrm{p}>.05]$ ).

El factor ético normativo hace referencia a individuos que reflejan los mandatos culturales por medio de valores sociopersonales aceptables; ellos se muestran menos pasivos, sin poder relajarse en sus actividades perceptivas, su actividad cognitiva se enfoca más en la concentración y el esfuerzo que en un simple dejarse ir; presenta un aplanamiento emocional, esperando de esto inhibiciones y preocupaciones (Color, $[\mathrm{r}=-.422, \mathrm{p}>$.05]). Tienen una habilidad creativa que les permite identificarse con la acción, son personas introvertidas que tienden a vivir en sí mismos (Movimiento, [ $\mathrm{r}=.378, \mathrm{p}>.05]$ ). Cuentan con la capacidad para llevar a cabo un esfuerzo intelectual, poseen un pensamiento realista, lógico y una organización cognitiva que les posibilita emplear su fantasía (Integración, $[\mathrm{r}=.383$, p>.05]). Son espontáneos (Animal, 
$[\mathrm{r}=.373, \mathrm{p}>.05])$, con pocas preocupaciones corporales (Anatomía, [r=.382, $\mathrm{p}>$.05]). Se consideran maduros, pero con preocupaciones sobre la vida sexual (Sexo, $[\mathrm{r}=.477, \mathrm{p}>.01]$ ).

El factor de inteligencia socioemocional se refiere a personas equilibradas, flexibles al enfrentar relaciones interpersonales y problemas de la vida, ellos se muestran sin una necesidad de autonomía (Espacio, [r=-.389, $\mathrm{p}>.05]$ ). Tienen una buena concentración y atención prolongada, y unen a su vez experiencia y memoria, empleando recursos creativos (Forma definida, $[\mathrm{r}=.401$, $\mathrm{p}>$.05]). Poseen un pensamiento realista, lógico y una organización cognitiva que les permite emplear su fantasía (Integración, $[\mathrm{r}=.527, \mathrm{p}>.01]$ ). Son espontáneos (Animal, [r=.549, p>.01]). Cuentan con la habilidad para comunicarse consigo mismos y con los demás, se adaptan al estrés, son resistentes a la sugestión (Barrera, [r=.433, $\mathrm{p}>.05]$ ).

El factor control externo negativopasivo se refiere a sujetos que cuentan con características negativas y demuestran una incapacidad y desinterés para actuar constructivamente; en el medio ellos se muestran -en relación con el HIT- como personas que viven en un mundo de fantasías, parcialmente alertas a los estímulos externos, con poco contacto con la realidad, lo cual está asociado con un bajo funcionamiento intelectual e inmadurez (Forma apropiada, $[\mathrm{r}=.404, \mathrm{p}>.05])$.
El factor emotivo negativo-autoafirmativo describe a personas con un temperamento negativo, normalmente se muestran inconformes, impulsivos y con una exaltación de las emociones; correlacionan en forma negativa con el HIT, lo que indica que son menos ansiosas, menos inseguras; cuentan con menos miedo y fobias y se muestran a sí mismas con más confianza (Ansiedad, $[\mathrm{r}=-.383, \mathrm{p}>.05])$.

El factor instrumental constructivo representa a personas que reflejan un funcionamiento adecuado y que cuentan con habilidades en diferentes ámbitos, como el trabajo, la escuela o el hogar; de igual modo, la correlación refleja que cuentan con una buena concentración y atención prolongada, con un sentido de responsabilidad para sí mismos como para con los demás (Forma definida, [r=.413, $\mathrm{p}>.05]$ ). Tienen menos incertidumbre, pueden dar estructura a estímulos ambiguos, son menos ansiosos, tienen una capacidad empática (Sombreado, [ $\mathrm{r}=-.371, \mathrm{p}>.05]$ ). Cuentan con una habilidad creativa que les permite identificarse con la acción, son personas introvertidas (Movimiento, $[\mathrm{r}=.450, \mathrm{p}>.05])$. Cuentan con la capacidad para llevar a cabo un esfuerzo intelectual, poseen un pensamiento realista, lógico y una organización cognitiva que les posibilita emplear su fantasía (Integración, [r=.438, p>.05]). Asimismo, se muestran espontáneos (Animal, $[\mathrm{r}=.548, \mathrm{p}>.01])$. 


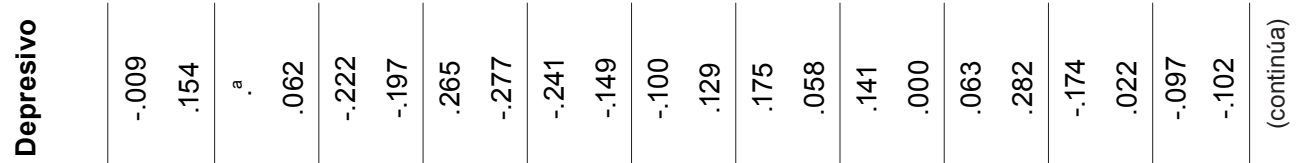

焉

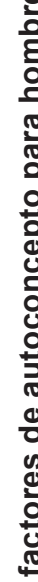

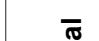

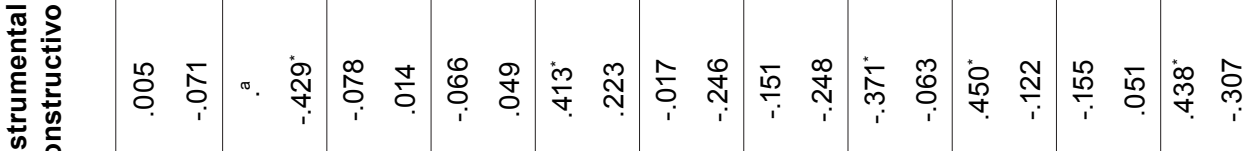

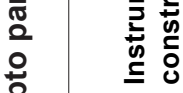

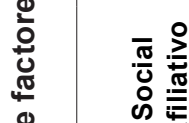

-

뜽응

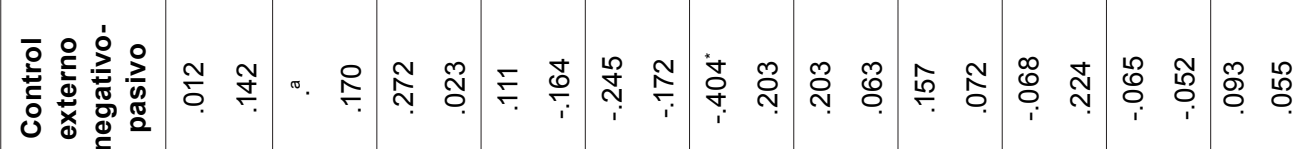

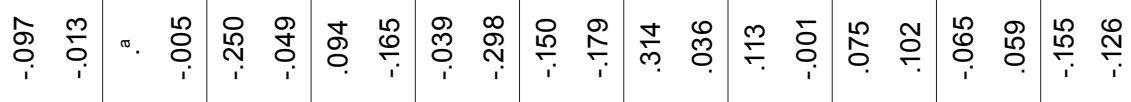

空

$\frac{\sqrt{2}}{\frac{9}{9}}$

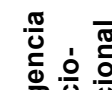

毵品高

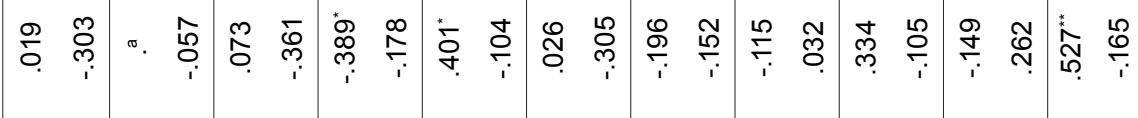



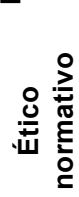


高产

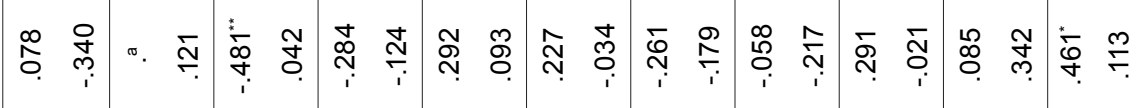

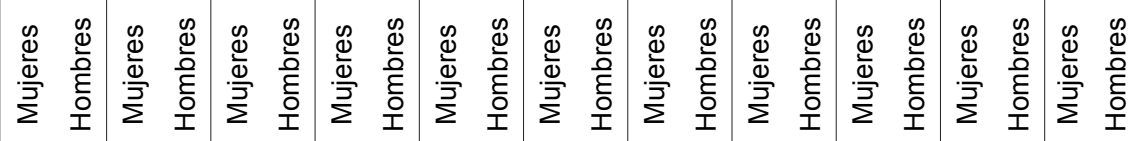

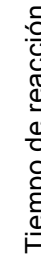

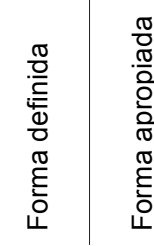

$\frac{5}{0}$

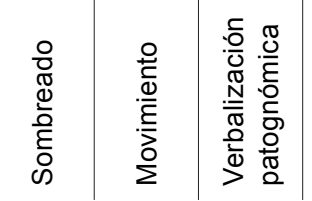

总 




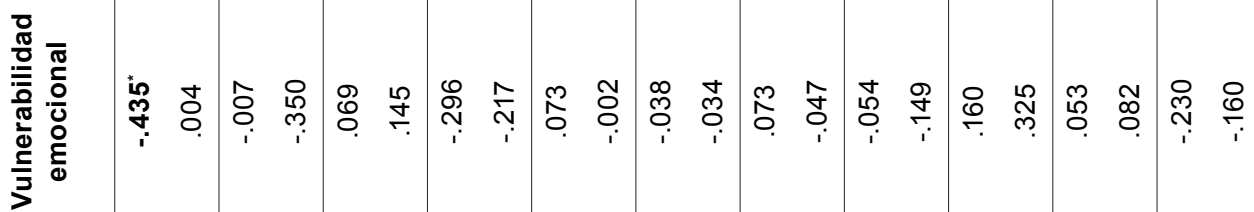

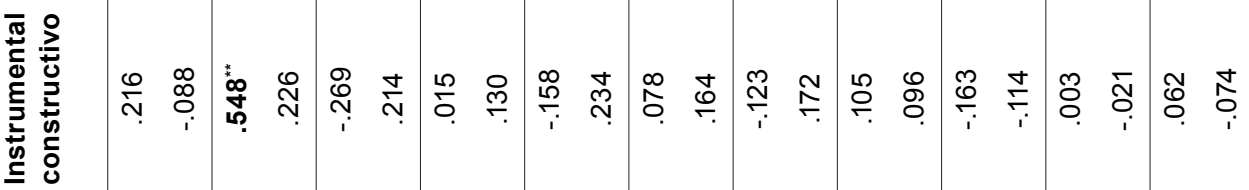

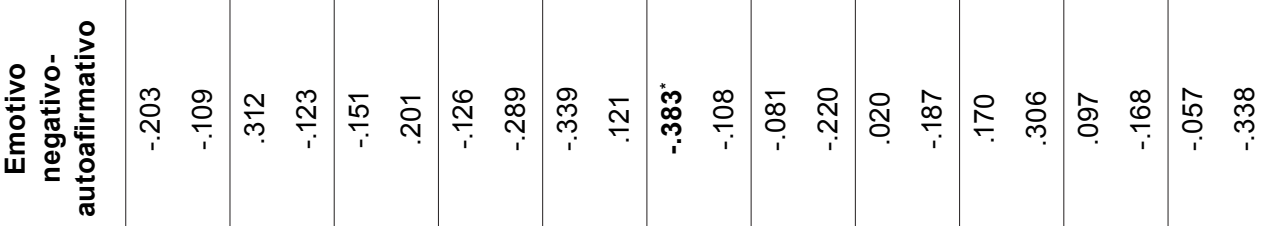

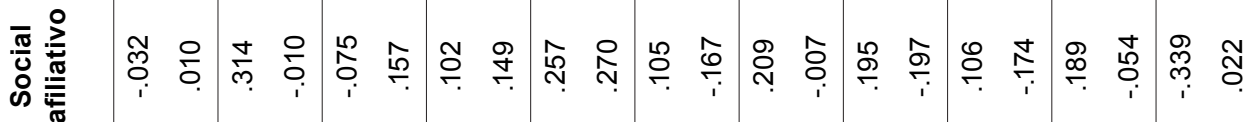

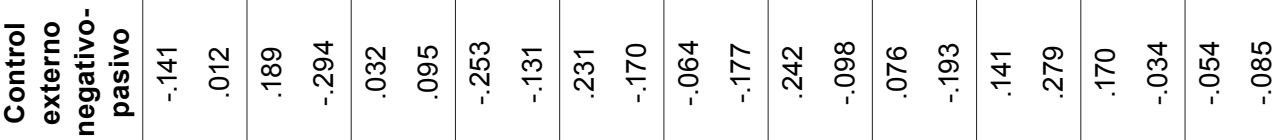

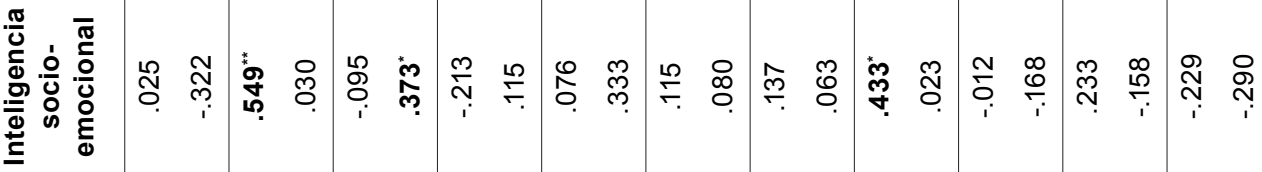
童产产

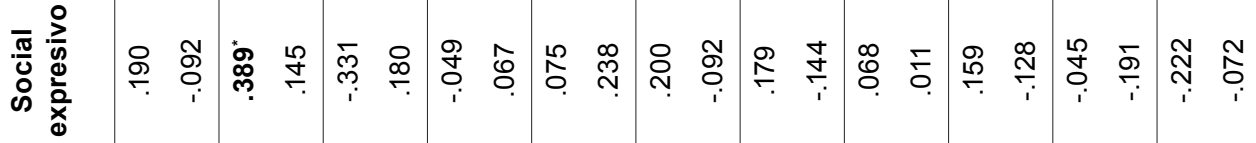

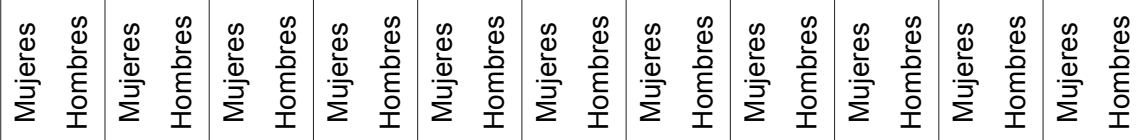

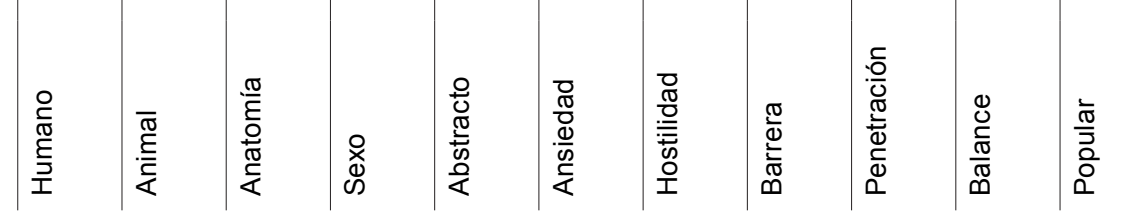


El factor instrumental constructivo representa a personas que reflejan un funcionamiento adecuado y que cuentan con habilidades en diferentes ámbitos, como el trabajo, la escuela o el hogar; de igual modo, la correlación refleja que cuentan con una buena concentración y atención prolongada, con un sentido de responsabilidad para consigo mismos como para con los demás (Forma definida, $[\mathrm{r}=.413, \mathrm{p}>.05]$ ). Tienen menos incertidumbre, pueden dar estructura a estímulos ambiguos, son menos ansiosos, tienen una capacidad empática (Sombreado, [ $\mathrm{r}=-.371, \mathrm{p}>.05]$ ). Entre sus cualidades está la habilidad creativa, que les permite identificarse con la acción, son personas introvertidas (Movimiento, $[\mathrm{r}=.450, \mathrm{p}>.05]$ ). Poseen la capacidad para llevar a cabo un esfuerzo intelectual, un pensamiento realista, lógico y una organización cognitiva que hace posible que empleen su fantasía (Integración, $[\mathrm{r}=.438, \mathrm{p}>.05]$ ). Asimismo, se muestran espontáneos (Animal, [r=.548, $\mathrm{p}>$.01]).

El factor de vulnerabilidad emocional hace referencia a las personas que cuentan con estados de ánimo negativo y se caracterizan por ser indiferentes, vulnerables y con disgustos; se muestran, en la correlación con el HIT, con poco interés en los demás, son poco cálidas y poco empáticas en sus relaciones interpersonales, y evidencian insensibilidad frente a los problemas de los otros (Humano, [r=.- 435, $\mathrm{p}>.05]$ ).
El factor depresivo describe a las personas que poseen características negativas, demuestran nostalgia, alteración y un deterioro en su salud mental; su correlación con el HIT es negativa, evidencian poco interés en los demás, son poco cálidas y poco empáticas en sus relaciones interpersonales, y muestran insensibilidad frente a los problemas de los otros (Humano, [r=-.445, $\mathrm{p}>.05]$ ).

\section{Correlaciones obtenidas para los hombres}

El factor instrumental se refiere a las personas que reflejan un funcionamiento adecuado y que cuentan con habilidades en diferentes hábitos, como el trabajo, la escuela o el hogar; se muestran en el HIT como personas colaboradoras para satisfacer las demandas, así como para realizar adecuadamente las tareas que se les solicita (Rechazo, [r=-.429, $\mathrm{p}>.05]$ ).

El segundo factor con el que correlacionan los hombres es el de inteligencia socioemocional, que evoca a las personas equilibradas, flexibles al enfrentar relaciones interpersonales y problemas de la vida; en su correlación con el HIT se muestran con preocupaciones corporales (Anatomía, [r=.373, $\mathrm{p}>.05]$ ).

\section{DISCUSIÓN}

Estos resultados concuerdan con los encontrados por Díaz-Loving, Rivera y Sánchez (2001), ya que si bien se habla 
de que en la cultura los hombres reciben un entrenamiento orientado hacia la instrumentalidad y las mujeres hacia un entrenamiento más expresivo, se perciben ya indicios de la emancipación y empoderamiento de la mujer, en la medida en que ellas no solo muestran características expresivas tanto positivas como negativas, sino que también puntúan más formas en atributos instrumentales en los que en el pasado puntuaban más alto los hombres.

Por lo tanto, podemos ubicar a un conjunto de mujeres con características expresivas deseables, como alegres, divertidas, animadas, sociables; con cualidades que les permiten una adecuada comunicación; flexibles al enfrentar relaciones interpersonales; honestas, decentes, leales, con buena concentración, así como con manejo de sus emociones.

Un conjunto aparte referente a las mujeres las muestra con características instrumentales positivas: ordenadas, trabajadoras, puntuales, activas, con una buena concentración, atención prolongada; además, cuentan con pensamiento lógico y un sentido de responsabilidad para consigo mismas como para con los demás.

Estas características, como refieren Parsons \& Bales (1955), hablan de una orientación expresiva y orientada a las relaciones interpersonales, conceptos inmersos dentro de la filosofía tradicional femenina, ya que cuentan con los atributos de comunión y empatía, características dirigidas a resolver los problemas del grupo social al que se pertenece y no solo al desarrollo del individuo.

Por otro lado, en las mujeres que manifiestan rasgos no deseables se puede encontrar que una parte de los resultados hacen referencia a aquellas con una expresividad negativa, a las que se considera indiferentes, vulnerables, amargadas, apáticas, solitarias, tímidas; con poco interés en los demás y poco cálidas. Estas mujeres muestran un deterioro en su salud mental, y poca empatía en sus relaciones interpersonales.

Otro grupo de mujeres con características instrumentales negativas muestran incapacidad y desinterés por actuar en su medio; son consideradas como ineptas, frustradas, falsas, pesimistas, conflictivas, temperamentales, enojonas; sin embargo, estas mismas se muestran con más confianza, pero con bajo funcionamiento intelectual e inmadurez.

Estos resultados concuerdan con los obtenidos por Díaz-Loving, Rivera y Sánchez (2001), los cuales describen características emocionales negativas que denotan debilidad y vulnerabilidad, vestigios de una construcción social que conceptualiza a la mujer como un ser dependiente y poco capaz de defenderse, con un espíritu contrario a lo comunal y empático, pues intenta resolver solo los problemas individuales y no los del grupo; características que deben 
considerarse negativas, en la medida en que impiden que una persona sea capaz de resolver problemas por sí misma, por lo que su desarrollo se ve truncado al necesitar siempre a alguien que lo salve.

Los hombres, por su parte, poseen características instrumentales deseables: ordenados, trabajadores, puntuales, activos, colaboradores para satisfacer las demandas en las tareas que se le solicita; asimismo, se muestran tranquilos, serenos y calmados.

Estos resultados difieren de los encontrados por Díaz-Loving, Rivera y Sánchez (2001). Los individuos no muestran características instrumentales de tipo individualista, sino características instrumentales deseables más para las mujeres, que les permiten un buen funcionamiento dentro del trabajo, la escuela; además de verlos como más colaboradores en las tareas y relaciones interpersonales. El hecho de que se muestren como tranquilos, serenos y calmados probablemente se deba a que presentan una preocupación corporal, porque tienen miedo a causarse daño.

Se debe recordar que es el proceso de socialización dentro de un espacio ecosistémico el que determina la forma como se percibe el mundo (Díaz-Loving, 2010). Es por medio del aprendizaje de estos patrones culturales que se asignan comportamientos y características adecuadas, ya sea para los hombres como para las mujeres (Rocha, 2000).
En este sentido, las diferencias en términos de la personalidad de un individuo, pese a que tienen una base genérica cromosomal, son un producto del intercambio social y de la interacción, por lo cual la caracterización es contextual, normativa, y está expuesta a un continuo proceso de trasformación (Díaz-Loving, Rocha \& Rivera, 2004).

Lo referido por los sujetos mediante la escala de autoconcepto recoge las características que los individuos consideran como propias y de las que ellos son conscientes, ya que las manifiestan mediante un autorreporte. Sin embargo, estas características se presentan en el individuo dentro de un contexto cultural, el cual tiene influencia sobre la base de ideas consensuadas o estereotipos. $\mathrm{Y}$ es por medio de las manchas de tinta que podemos apreciar la manifestación de lo introyectado, de lo inconsciente, de lo aprendido culturalmente, de lo basado en ideas consensuadas o estereotipos, pero no manifestado mediante la conciencia sino mediante su percepción. La técnica de las manchas de tinta recoge estos procesos de percepción asignados a cada individuo dentro de su propia cultura; si bien las manchas se valen de un proceso de proyección los individuos proyectan -valga la redundancia- su Yo, el cual es una estructura estable a través del tiempo, y se construye en su actuar y en su interacción con el medio ambiente. De esta manera, la respuesta interna está permeada por 
su propia experiencia y también por los procesos culturales a los que ha estado sometido.

Así, el proceso de socialización no solo entreteje los rasgos de los individuos sino que pone de manifiesto su papel como mediador y constructor de nuestra personalidad, delimitando la percepción a lo asignado a cada uno de los sexos. A su vez, la percepción que se tiene del mundo estructura la personalidad, volviéndose un bucle de retroalimentación.

Los resultados obtenidos por medio de la correlación de la escala de autoconcepto y del HIT reflejan la intensidad de los rasgos, los que no solo son referidos por el sujeto de una manera abierta sino que son corroborados por la misma forma de percepción. Se puede dar que dentro de esta posible construcción un sujeto manifieste como características propias elementos que ha internalizado y que ha podido hacer conscientes a lo largo de este aprendizaje cultural y de su vivir diario.

Esta manifestación de lo inconsciente y lo consciente no debe verse como elementos independientes que constituyen de una u otra forma un mismo individuo, sino como elementos que conforman la totalidad del sujeto; y la forma de acceder a este contenido es la que se da por caminos diferentes. Sin embargo, conviene considerar que si bien la vía proyectiva posiblemente brinde información a la que no se tiene tanto acceso, es necesario un procedimiento que cuente con la metodología y el sustento científico necesario para extraer los contenidos con la mayor validez y confiabilidad posibles.

A pesar de que hoy en día los hombres y las mujeres son partícipes de importantes transformaciones individuales, sociales y culturales, que cuestionan la exclusividad con la que se han impuesto comportamientos y tareas, diferentes y excluyentes, entre los géneros, sigue existiendo una resistencia social e individual al rompimiento de la polarización de género, lo que constituye una de las barreras psicológicas, sociales y culturales más fuertes en el proceso de una transformación verídica de identidades (Rocha \& Díaz-Loving, 2011).

Por último, cabe mencionar que la interpretación de las manchas de tinta debe verse también como un ejercicio para conocer las variaciones de percepción, en la medida en que estas son contextuales dentro de cada cultura y no solo un ejercicio de introspección.

\section{REFERENCIAS}

Bem, S. (1981). Gender schema theory: A cognitive account of sex typing. Psychology Review, 88, 354-364.

Bem, S. (1993). The lenses of gender: transforming the debate on sexual inequality. New Haven, CT: Yale University Press. 
Cattell, R. (1972). Análisis científco de la personalidad. Barcelona: Fontanella.

Codina, N. (2005). El self y sus pluralidades: un análisis desde el paradigma de la complejidad. Escritos de Psicología, 7, 24-34.

Díaz-Guerrero, R. (2003). Bajo las garras de la cultura. México, D. F.: Trillas.

Díaz-Guerrero, R., \& Díaz-Loving, R. (1991). Introducción a la psicología: Un enfoque sistémico. México, D. F.: Trillas.

Díaz-Loving, R. (2010). Los mexicanos. Conducta, 8(16), 75-85.

Díaz-Loving, R., Reyes Lagunes, I. \& Rivera Aragón, S. (2002). Autoconcepto: Desarrollo y validación de un inventario etnopsicológico. Revista Iberoamericana de Diagnóstico y Evaluación Psicológica, 13, 1, 29-54.

Díaz-Loving, R., Rivera, A., \& Sánchez, R. (2001). Rasgos instrumentales (masculinos) y expresivos (femeninos), normativos (típicos e ideales) en México. Revista Latinoamericana de Psicología, 33(2), 131-139.

Díaz-Loving, R., Rivera, S., \& Sánchez, R. (2010). Género y pareja. En: Díaz-Loving, R., \& Rivera, S. (comps.). Antología. Psicología de la pareja, clásicos y contemporáneos (215-242). México, D. F.: Porrúa/
Universidad Nacional Autónoma de México.

Díaz-Loving, R., Rocha, S. T., \& Rivera, S. (2004). Elaboración, validación y estandarización de un inventario para evaluar las dimensiones atributivas de instrumentalidad y expresividad. Revista Interamericana de Psicología, 38(2), 263-276.

González, M. (2006). Las manchas de tinta / The ink spots. Revista del Hospital Psiquiátrico de La Habana, 3 (3). (Recuperado el 3 de febrero del 2011, de <http://www.revistahph.sld.cu/>.

Hill, E. F. (1972). The Holtzman inkblot technique: a Handbook for clinical application. Vargas, J. \& Villagómez, I., (eds. y trads.). En: Vargas, J., \& Villagomez, I. (2003). Estudio preliminar a la estandarización de la técnica de manchas de tinta de Holtzman en estudiantes universitarios. Tesis de licenciatura. México, D. F.: Universidad Nacional Autónoma de México.

Holtzman, W. H., Thorpe, J. S., Swartz, J. D., \& Herron, E. W. (1971). Interpretación de manchas de tinta, técnica Holtzman. México, D. F.: Trillas.

Matesanz, A. (1997). Evaluación estructurada de la personalidad. Madrid: Pirámide.

Padilla-Bautista, J. A. (2012). Rasgos de personalidad mediante la técnica 
de manchas de tinta de Holtzman en hombres y mujeres estudiantes de psicología. Tesis de licenciatura. México, D. F.: Universidad Insurgentes.

Parsons, T. \& Bales, R. F. (1955). Family socialization and interaction process. Glencoe: Free Press.

Rocha, S. T. (2000). Roles de género en los adolescentes mexicanos y rasgos de masculinidad-feminidad. Tesis de licenciatura. México, D. F.: Universidad Nacional Autónoma de México, Facultad de Psicología.

Rocha, T., \& Díaz-Loving, R. (2011). Identidades de género (Más allá de cuerpos y mitos). México, D. F.: Trillas.

Shaffer, D. (2000). Psicología del desarrollo: infancia y adolescencia. México, D. F.: Internacional Thomson.

Tamayo, A. (1982). Autoconcepto, sexo y estado civil. Revista de la Asociación Latinoamericana de Psicología Social, 2, 3-15.

Vargas, J., \& Villagómez, I. (2003). Estudio preliminar a la estandarización de la técnica de manchas de tinta de Holtzman en estudiantes universitarios. Tesis de licenciatura. México, D. F.: Universidad Nacional Autónoma de México. 